\title{
Pronóstico de la injuria renal aguda en el corto y largo plazo
}

\author{
Short and long term prognosis of acute kidney injury \\ Javier Cieza ${ }^{1, a, b ; 2, c}$, Juan Lluncor 1,2,d, Augusto Cama 2,e

\section{RESUMEN}

La injuria renal aguda (IRA) adquirida en la comunidad puede tener pronóstico diferente a largo plazo que la intrahospitalaria, situación escasamente descrita. Objetivos: Comparar la supervivencia a largo plazo de pacientes con IRA con pacientes que no la tuvieron al ingresar al hospital y con pacientes en hemodiálisis crónica (HD). Material y métodos: Se estudiaron pacientes con y sin IRA diagnosticada al momento de ser admitidos a la emergencia del Hospital Cayetano Heredia $(\mathrm{HCH})$ y se comparó la supervivencia mediante curvas de Kaplan-Meier y análisis multivariado de Cox. Se comparó también con sus contemporáneos que ingresaron a HD. El período de observación fue entre diciembre del 2011 y el 31 de diciembre del 2015. Resultados: Fueron estudiados 47 pacientes con IRA, 93 sin IRA y 138 en HD. Las tasas de letalidad de los pacientes con y sin IRA no alcanzaron diferencia significativa a los tres $(p=0,148)$ ni a los 35 meses $(p=0,068)$. Hubo diferencia al comparar las tasa de letalidad entre los pacientes con IRA con los pacientes en HD ( $\mathrm{p}=0,0002)$, pero ésta desapareció al ser normalizados los grupos por edad, albúmina sérica y ausencia de neoplasia maligna $(\mathrm{NM})(\mathrm{p}=0,177)$. En el análisis multivariado de Cox, las variables que afectaron la supervivencia fueron: edad avanzada $(p=0,001)$, hipoalbuminemia severa $(p=0,001)$ y coexistencia de NM ( $p=0,03)$. Conclusiones: La IRA adquirida en la comunidad no tiene efecto per se en la supervivencia de los pacientes a largo plazo. Esta es dependiente de la edad, la desnutrición y la coexistencia de NM.

PALABRAS CLAVE: Insuficiencia renal, pronóstico, diálisis renal. (Fuente: DeCS BIREME).

\section{SUMMARY}

Community acquired acute kidney injury (AKI) may have a different long-term prognosis than hospital acquired AKI. Objectives: To compare long-term survival of patients with AKI with that of patients without AKI and with that of patients on chronic hemodialysis (HD). Methods: Patients with and without AKI diagnosed at admission were included, survival analysis using Kaplan-Meir with multivariate Cox proportional methods were used. A comparator group of patients on HD was also included in the analysis. The study covered the period between December 2011 and 31 December 2015. Results: A total of 47 patients with AKI, 93 without AKI and 138 in HD were included. There was no statistical difference in case fatality rates between patients with and without AKI at $3(p=0.148)$ and 35 months $(p=0.068)$. There was difference in case fatality rate between patients with AKI and HD ( $p=0.0002)$,

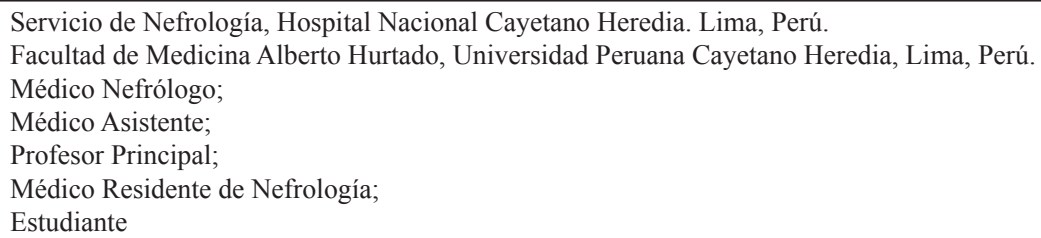


but this difference disappeared after adjusting for age, serum albumin and lack of cancer $(\mathrm{p}=0.177)$. Variables that affected mortality in the multivariate analysis were advanced age $(\mathrm{p}=0.001)$, severe hypoalbuminemia $(\mathrm{p}=0.001)$ and concomitant cancer $(\mathrm{p}=0.03)$. Conclusions: Community acquired AKI has no effect on long-term survival of patients, which depends on age, malnutrition and concomitant cancer.

KEY WORDS: Renal insufficiency, prognosis, renal dialysis. (Source: MeSH NLM).

\section{INTRODUCCIÓN}

La injuria renal aguda (IRA) es un proceso mórbido y grave en la pérdida de la salud de las personas, que en lo posible no debería ser visto en forma aislada del contexto general del paciente $(1,2)$. El estudio de Lluncor et al, (3) muestra con claridad aspectos de los pacientes que los hacen vulnerables para desarrollar la IRA en nuestra comunidad y que no habían sido enfatizados en otras publicaciones porque muchas veces se subestiman o porque los estudios comparables han sido realizados en poblaciones bastante diferentes a la estudiada por estos autores (4-8). Subrayaremos la coexistencia de enfermedades neuro-psiquiátricas, la presencia de enfermedad respiratoria o cáncer y el consumo crónico de tabaco y alcohol. Es decir, los pacientes incidentes a nuestros hospitales con pérdida aguda de la función renal, son personas biológicamente más vulnerables que los que ingresan $\sin \operatorname{IRA}(2,3)$.

No es entonces sorprendente que los pacientes con IRA lleguen a los hospitales más graves, infectados o en septicemia (3,9-11), lo que condiciona un mayor riesgo de muerte en el corto plazo el cual no se modifica aun con intervenciones médicas enérgicas, idóneas y oportunas como está ampliamente descrito en la literatura mundial, salvo reportes aislados $(4,9,10)$. Estas intervenciones en el reemplazo de la función renal perdida, han sido y son la diálisis continua, hemodiálisis convencional o de alto flujo, la hemofiltración o la hemodiafiltración, que salvo reportes aislados, no han mostrado mayor éxito en cambiar el pronóstico final del paciente dentro del hospital. El pronóstico en el corto plazo es pues malo (12).

Un segundo aspecto a resaltar es el relacionado al daño renal permanente en el que quedan muchos de estos pacientes y que con el tiempo, genera los efectos propios del daño crónico de la función renal. Este efecto se ha objetivado en la mayor letalidad que el esperable en el largo plazo que aquel dependiente solo del daño renal (13).
En este sentido, este estudio tratará de responder dos cuestiones, ¿tienen los pacientes con IRA mayor riesgo de muerte en el largo plazo que sus pares incidentes en la misma época, edad y sexo pero que no tuvieron IRA? y en segundo lugar, ¿tienen mayor letalidad los pacientes incidentes con IRA que aquellos incidentes con Enfermedad Renal Crónica terminal en el mismo período de tiempo?

\section{MATERIAL Y MÉTODOS}

Estudio observacional de cohortes concurrentes de pacientes adultos incidentes al Departamento de Emergencia del Hospital Cayetano Heredia en los meses de noviembre y diciembre del 2011 expuestos y no expuestos a IRA, apareados en el tiempo de admisión (mismo día) y mismas condiciones al ser admitidos. Aceptando una tasa anual de muerte promedio del $10 \%$ tal cual ocurre en los pacientes con enfermedad renal crónica termina (ERCt) en hemodiálisis (30\% en tres años) y considerando dos pacientes no expuestos por uno expuesto, una confianza del $95 \%$, una potencia del $80 \%$ y un riesgo relativo mínimo diferencial de 1,5 se determinó un tamaño de muestra que para un seguimiento a tres años fue de al menos 36 pacientes expuestos y 72 no expuestos.

Se consideró el documento nacional de identidad (DNI) del Perú referido por el paciente o familiares directos para realizar el seguimiento según el padrón de registro de los ciudadanos peruanos (RENIEC) y definir la situación de estar vivo o muerto al 31 de diciembre del 2015.

Se registró la edad para definir el grupo etario (entre 18 y 64 años, entre 65 y 81 años y mayores de 81 años), sexo y las situaciones de comorbilidades clínicas relevantes de todos los pacientes al momento de la admisión (gravedad expresada en el score SOFA, estado de shock, estado de sepsis, diagnóstico de enfermedad infecciosa al ingreso, existencia o no de hipertensión arterial, diabetes mellitus, accidente cerebrovascular, enfermedad cardiovascular, enfermedad respiratoria, tuberculosis, infección 
por VIH, neoplasia maligna, consumo de alcohol y tabaco, enfermedad neuropsiquiátrica y enfermedad inmunológica). Se consignaron y estratificaron los siguientes datos de laboratorio al momento de la admisión: urea (urea menor de $40 \mathrm{mg} / \mathrm{dl}$, entre $21 \mathrm{y}$ $40 \mathrm{mg} / \mathrm{dl}$ y más de $40 \mathrm{mg} / \mathrm{dl}$ ), creatinina (menor de 1,1 $\mathrm{mg} / \mathrm{dl}$ y más de $1,1 \mathrm{mg} / \mathrm{dl}$ ) y nivel de albúmina sérica (menos de 2,6 g/dl, entre 2,6 y 3,5 g/dl y más de 3,5 $\mathrm{g} / \mathrm{dl}$ ). Se excluyeron los pacientes que no tuvieran al menos tres determinaciones de creatinina sérica en su evolución intrahospitalaria o que no aceptaron firmar el consentimiento informado.

Para la comparación de la supervivencia de los pacientes con IRA y la de pacientes con ERCt en terapia de reemplazo renal crónica, se seleccionaron con el permiso pertinente, los pacientes incidentes al programa de hemodiálisis crónica de la Universidad Peruana Cayetano Heredia provenientes del Hospital Cayetano Heredia que hubieren ingresado a partir de enero del 2011 y que hubieran permanecido más de tres meses en el programa. Identificados los pacientes se procedió a constatar su situación de estar vivo o haber fallecido hasta el 31 de diciembre del año 2015. De estos pacientes se registraron las variables de edad y sexo.

Posteriormente, se estudió la supervivencia de los pacientes con IRA contra sus controles sin IRA incidentes al hospital para definir si había diferencia y determinar el efecto de las comorbilidades existentes. Luego se comparó la supervivencia de los pacientes con IRA contra los pacientes ingresados al programa de hemodiálisis crónica desde el año 2011 provenientes del mismo hospital.

Para el trato estadístico se utilizó las curvas de supervivencia de Kaplan-Meier y el análisis de regresión de Cox para definir las variables fundamentales implicadas en la supervivencia. El software utilizado fue el SPSS v.18 y se aceptó como nivel de significancia estadística el valor $\mathrm{p}<0,05$.

\section{RESULTADOS}

Se estudiaron 47 pacientes incidentes con IRA a la emergencia del $\mathrm{HCH}$ en el período entre noviembre y diciembre del año 2011 (Grupo IRA) y 91 pacientes del mismo periodo de estudio incidentes a la misma emergencia sin IRA (Grupo no-IRA). Se incluyeron 101 pacientes con ERCt que ingresaron al programa regular de hemodiálisis crónica (Grupo HD) desde el 1 de enero del 2011 y que se mantuvieron al menos tres meses en el programa.

Letalidad general y en los primeros tres meses de los Grupos IRA y no-IRA

En la tabla 1 se presentan las tasas de letalidad de los pacientes con y sin IRA a los tres meses y al final del estudio, no encontrándose diferencia estadísticamente significativa entre los dos grupos de estudio. En el gráfico 1 se presenta la supervivencia general de los pacientes con y sin IRA.

En la tabla 2 se muestra los resultados de la supervivencia de los pacientes de los Grupos IRA y noIRA mediante la regresión de Cox en su modelo final. El pertenecer o no pertenecer al grupo IRA no presentó significancia estadística en el modelo final. Las variables halladas en este modelo fueron las mismas que alcanzaron significancia estadística en el modelo de regresión logística binaria cuando se consideraron todas las variables incluidas para el estudio en función de estar vivo o muerto. Estas variables fueron: el grupo etario (a mayor edad mayor letalidad) (Gráfico 2); la existencia de neoplasia maligna (Gráfico 3) y el tener un nivel de albúmina sérica igual o debajo de $2,5 \mathrm{mg} /$ dl (Gráfico 4).

Para comparar la supervivencia de los pacientes con IRA contra los que ingresaron a un programa regular de hemodiálisis crónica (HD) se normalizaron los grupos según los siguientes criterios: Edad: se eliminó a los mayores de 81 años (asociados a la

Tabla 1. Tasa bruta de letalidad de los pacientes con IRA y sin IRA.

\begin{tabular}{lccc}
\hline & Grupo IRA & Grupo No-IRA & $\boldsymbol{p}$ \\
\hline Situación a los tres meses & & 0,145 \\
$\quad$ vivos & $25(53,2 \%)$ & $61(65,9 \%)$ & \\
$\quad$ muertos & $22(46,8 \%)$ & $30(34,1 \%)$ & \\
Situación al final del estudio (media 35 m) & & & 0,068 \\
$\quad$ vivos & $24(51,1 \%)$ & $61(67,0 \%)$ & \\
$\quad$ muertos & $23(48,9 \%)$ & $30(33,0 \%)$ & \\
\hline
\end{tabular}




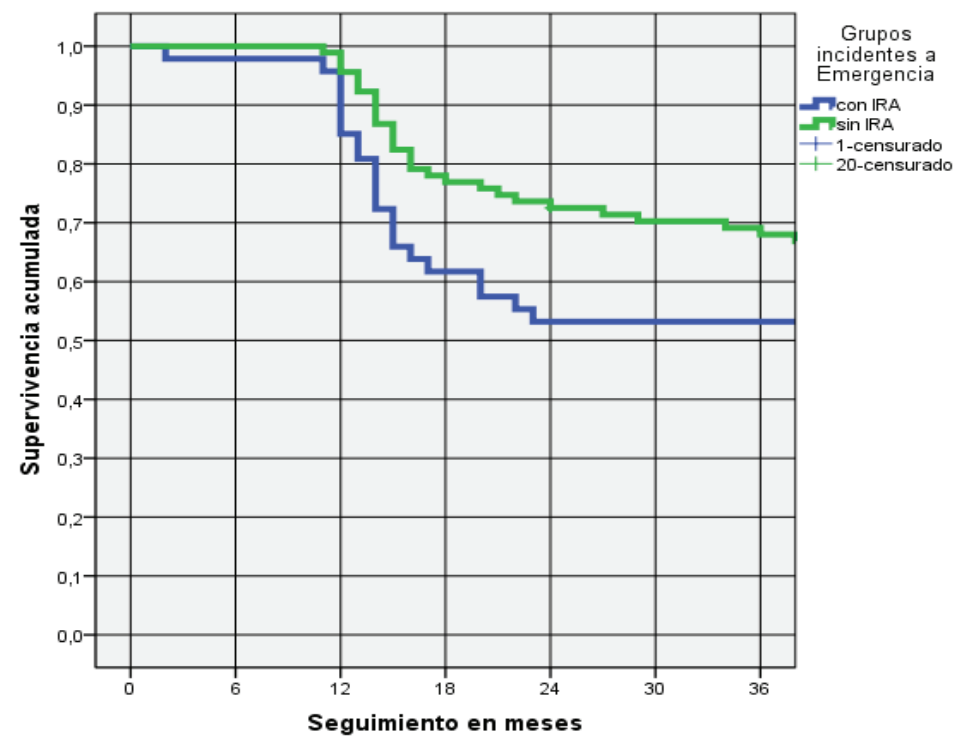

Gráfico 1. Supervivencia acumulada a los tres años de pacientes incidentes a emergencia con y $\sin$ IRA sin modelos de ajuste por edad, albúmina sérica y coexistencia de neoplasia maligna.

Tabla 2. Modelo final de supervivencia de los Grupos IRA y no-IRA y las variables influyentes en la letalidad final.

\begin{tabular}{lccc}
\hline Variable & Coeficiente B \pm EE & Exp. B & $\boldsymbol{p}$ \\
\hline Grupos etarios & $1,008 \pm 0,306$ & 2,740 & 0,001 \\
Presencia de neoplasia maligna & $2,564 \pm 1,180$ & 12,986 & 0,030 \\
Albúmina sérica según estratos & $-1,033 \pm 0,317$ & 3,560 & 0,001 \\
\hline
\end{tabular}

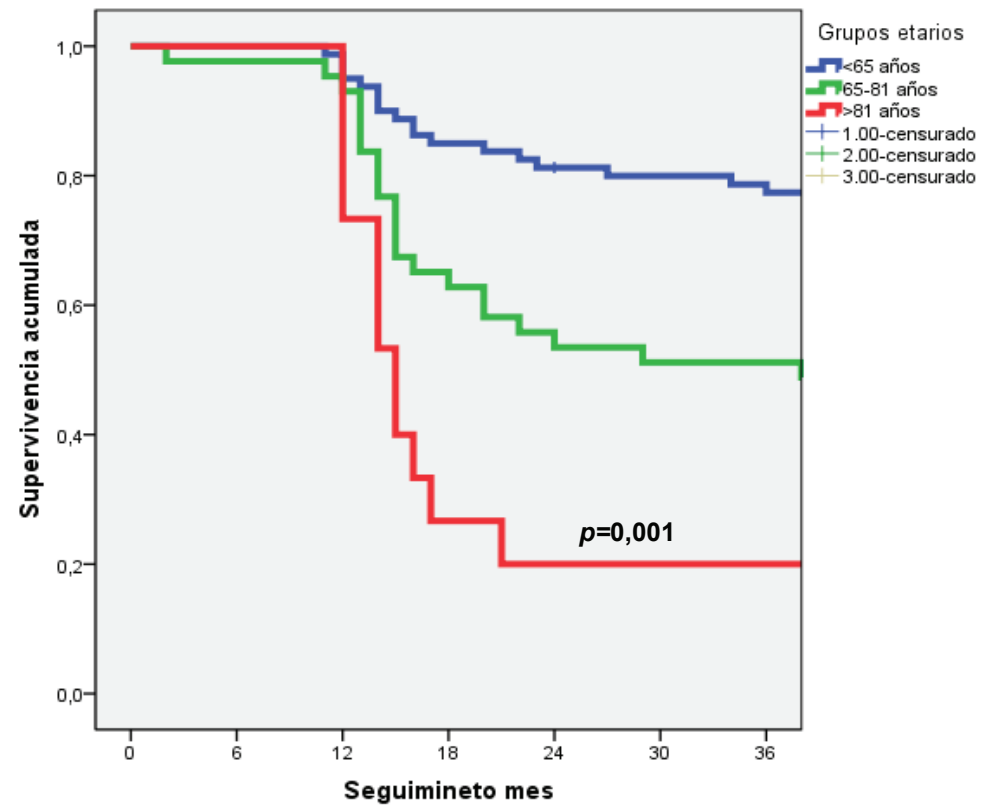

Gráfico 2. Supervivencia acumulada de pacientes con y sin injuria renal aguda en función de los grupos etarios. 


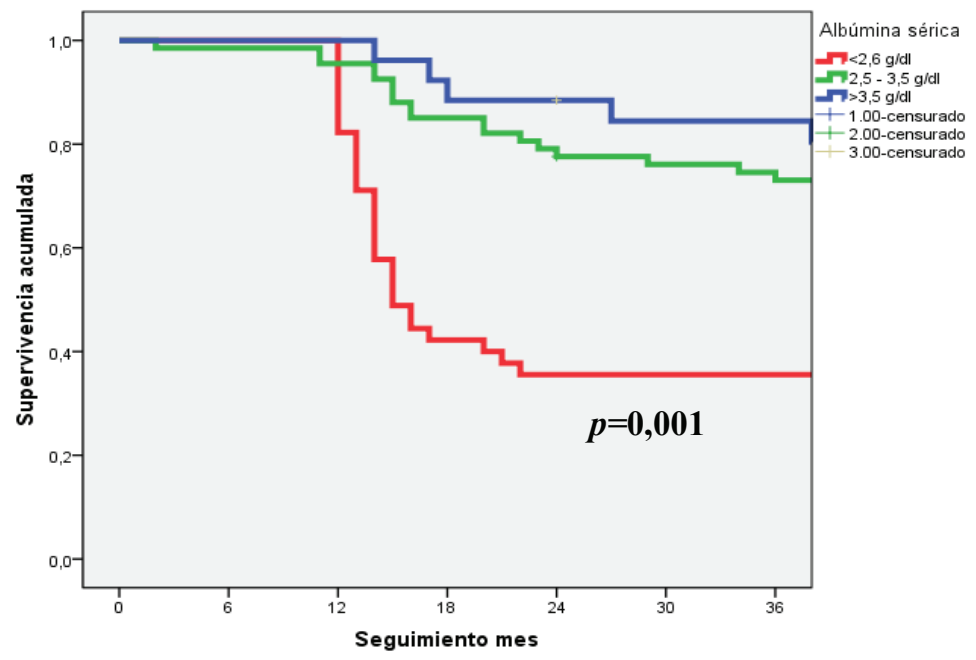

Gráfico 3. Supervivencia acumulada de pacientes con y sin injuria renal aguda en función del nivel de la albúmina sérica.

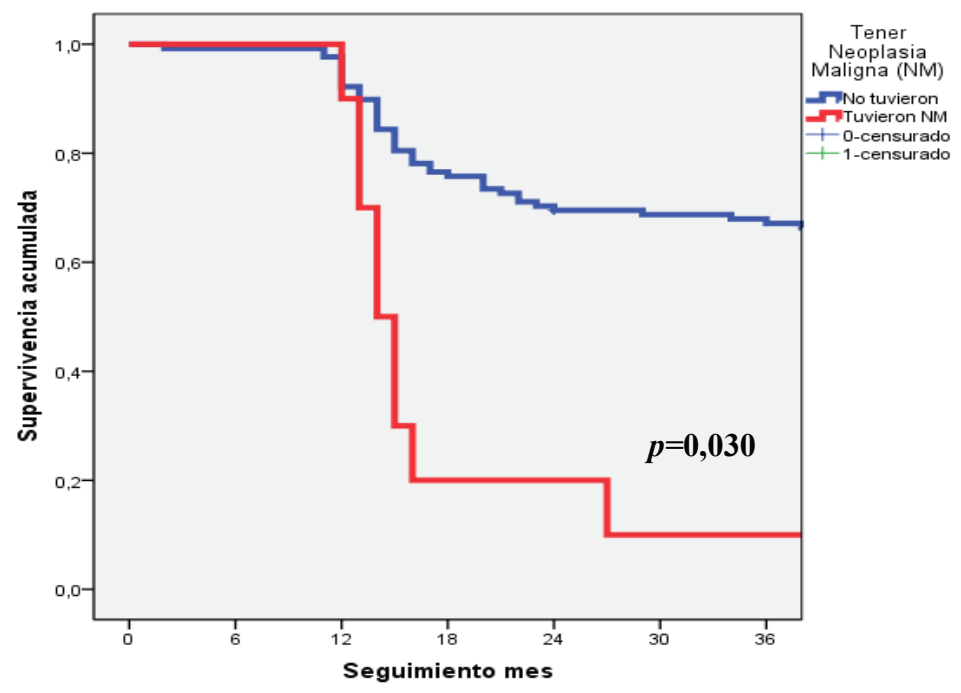

Gráfico 4. Supervivencia acumulada de pacientes con y sin injuria renal aguda en función de la coexistencia de neoplasia maligna.

Tabla 3. Tasa bruta de letalidad de los pacientes con IRA y en HD.

\begin{tabular}{|c|c|c|c|}
\hline & Grupo IRA & Grupo HD & $P$ \\
\hline Sin normalizar variables & & & 0,0002 \\
\hline vivos & $24(51,1 \%)$ & $81(80,2 \%)$ & \\
\hline muertos & $23(48,9 \%)$ & $20(19,8 \%)$ & \\
\hline TOTAL & $47(100 \%)$ & $101(100 \%)$ & \\
\hline $\begin{array}{l}\text { Normalizando edad, albúmina sérica y ausencia de } \\
\text { neoplasia maligna }\end{array}$ & & & 0,177 \\
\hline vivos & $15(65,2 \%)$ & $67(78,8 \%)$ & \\
\hline muertos & $8(34,8 \%)$ & $18(21,2 \%)$ & \\
\hline TOTAL & $23(100 \%)$ & $85(100 \%)$ & \\
\hline
\end{tabular}




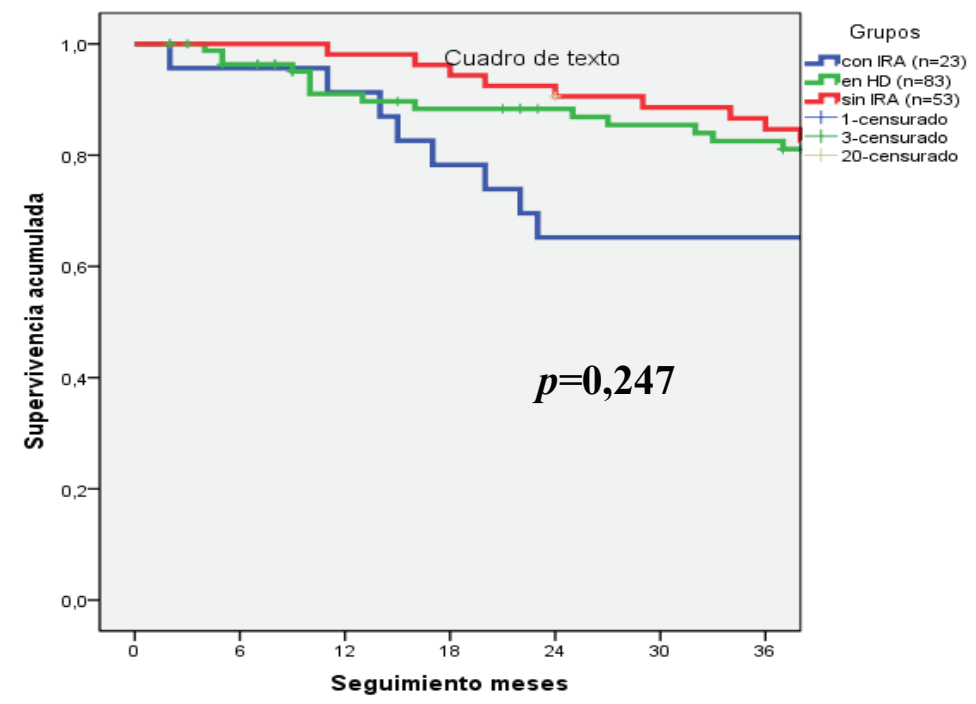

Gráfico 5. Supervivencia acumulada de pacientes con IRA, sin IRA y con enfermedad renal crónica terminal en programa de hemodiálisis crónica (HD) normalizada por el nivel de albúmina, inexistencia de neoplasia maligna y edad.

mayor letalidad en los grupos con IRA y sin IRA) y a los menores de 25 años (ausentes en los grupos con IRA y sin IRA).También se eliminaron a los que tuvieron coexistencia de neoplasia maligna (NM) y un valor de albúmina sérica igual o inferior a $2,5 \mathrm{mg} / \mathrm{dl}$.

Al comparar la tasa de letalidad entre el grupo con IRAy en HD se observó una diferencia estadísticamente significativa $(\mathrm{p}=0,0002)$ (Tabla 3) que desapareció $(\mathrm{p}=0,177)$ al normalizar los pacientes según la edad, nivel de albúmina y ausencia de NM.

El análisis de los datos normalizados, mostró que la comparación de las tasas de letalidad entre los grupos con IRA, sin IRA y los pacientes en HD, no alcanzó significancia estadística $(\mathrm{p}=0,221)$, así como tampoco la supervivencia vista en las curvas de Kaplan Meier $(\mathrm{p}=0,247)$ (Gráfico 5). El análisis multivariado de Cox tampoco mostró significancia estadística en ningún modelo ejecutado mediante el análisis paso a paso, siendo el óptimo resultado el que incluyó las variables grupos etarios y categoría de pacientes (grupo IRA, grupo no IRA y en $\mathrm{HD})(\mathrm{p}=0,063)$, aunque en este modelo la edad mostró ser la única variable relevante pero sin alcanzar significancia estadística por si misma $(\mathrm{p}=0,052)$.

\section{DISCUSIÓN}

El estudio que tuvo como objetivo determinar el efecto en la letalidad a largo plazo luego de haber tenido un evento intercurrente de IRA en comparación con quienes no lo tuvieron, mostró que el evento por sí mismo no afecta la letalidad y que las diferencias en las curvas de supervivencia ocurrieron por la edad avanzada del paciente (mayor de 81 años), la coexistencia de neoplasia maligna y el estar severamente desnutrido (visto a través de niveles de albúmina sérica igual o inferiores a los $2,5 \mathrm{mg} / \mathrm{dl}$ ).

En este punto resulta interesante apreciar la supervivencia que se encontrarían sin análisis multivariado, donde podría inducirse que la IRA tiene en sí misma una disminución en la supervivencia (Gráfico 1). De apreciar solamente la curva relativa al haber tenido IRA, se podría suponer que a los tres años la supervivencia es de $53 \%$, un valor relativamente bajo para la supervivencia si ésta se comparara con la reconocida para pacientes en programa crónico de diálisis de cualquier modalidad que es notablemente superior $(>60 \%$ a los cinco años) e induciría a extrapolar un pobre pronóstico de la IRA.

Al comparar la probabilidad de supervivencia de los pacientes que tuvieron IRA con la de los pacientes de la misma época que ingresaron a programa de HD regular crónica tampoco se halló diferencia estadísticamente significativa entre ambos cuando fueron normalizados en edad, nivel de albúmina sérica e inexistencia de neoplasia maligna, es decir, cuando las variables halladas como críticas en la supervivencia de pacientes que son admitidos al hospital con o sin 
IRA fueron eliminadas. Se excluyó para esta fase del análisis los pacientes adultos mayores frágiles (mayores de 81 años), los severamente desnutridos y los pacientes que tenían neoplasia maligna por haber previamente demostrado la importancia de éstas como variables de confusión. Es importante subrayar que todos los grupos provinieron de un mismo estrato social y fueron atendidos en la misma época y por el mismo proveedor de salud, situación que controla las variables propias de los hábitos y costumbres de los pacientes como adherencia a los tratamientos y efectos de la oferta de los servicios.

Nuestros resultados nos conducen a una conclusión que creemos muy válida, esta es, que la IRA en sí misma no afecta la supervivencia a largo plazo como se ha resaltado en otros estudios, sino que son las condiciones de vulnerabilidad biológica del paciente las que en el corto y largo plazo afectan su supervivencia.

$\mathrm{Al}$ respecto el trabajo de Lluncor et al (3), mostró que la IRA adquirida en la comunidad e incidente a la emergencia del Hospital Cayetano Heredia, estuvo asociada fuertemente a las condiciones de los pacientes como la coexistencia de enfermedad respiratoria, el consumo de alcohol y tabaco, la coexistencia de neoplasia maligna y la existencia de enfermedad neuropsiquiátrica y que estos pacientes llegaban más graves, generalmente sépticos o ingresaban a la emergencia con un cuadro infeccioso presente, situaciones a su vez más frecuentes en los pacientes desnutridos, aunque en esta referencia la desnutrición y la edad no tuvieron fuerza de asociación a la existencia o no de IRA en el modelos de casos y controles.

Sin embargo, en la supervivencia a largo plazo, la edad es una variable muy relevante y potente para los efectos estadísticos, sobre todo cuando ésta sobrepasa los 81 años. Este proceso que es un efecto biológico intrínseco de la vida, probablemente es el más relevante y muy difícil de controlar, pues depende fuertemente del proceso de envejecimiento saludable que en nuestras poblaciones es limitado $(7,8,14)$, básicamente por los problemas de nutrición que es la otra variable fuertemente ligada a la supervivencia y que podría no ser únicamente función del estado socioeconómico de la población, sino también de aspectos relacionados a los hábitos y costumbres alimentarios tenidos durante la vida $(9,15)$. En nuestra opinión, nuestra población es muy proclive a elevado consumo de carbohidratos y grasas e independiente de su estado socioeconómico el consumo de proteínas de alto valor biológico no es relevante, situación que se refleja en los elevado índices de sobrepeso u obesidad vistos en nuestras poblaciones que se aprecia aún en la infancia (16).

La tercera variable hallada relacionada a mayor letalidad es la coexistencia de neoplasia maligna, situación previsible para cualquier disfunción mayor del organismo y que resultaría innecesario profundizar más, excepto el hecho que esta comorbilidad tiene efectos potentes en el estado de desnutrición de las personas y que probablemente por ello aparece con claridad en el modelo de supervivencia $(17,18)$.

Nuestro estudio tiene la potencia estadística para soportar estas conclusiones porque el tamaño de la muestra para comparar riesgos ha sido el previsto en el tamaño muestral, excepto para la comparación con el grupo de pacientes que ingresaron en la misma época al programa de hemodiálisis crónica regular dado que se hizo el ajuste de las variables de confusión cuyo objetivo fue evitar el sesgo de análisis esperable si se la incluía. Aún con esta limitación, la diferencia estadística solamente dependiente de la injuria renal aguda o la enfermedad renal crónica estuvo bastante lejos de ser significativa $(\mathrm{p}=0,247)$ (Gráfico 5) y en el análisis multivariado de Cox, el grupo etario quedó marginalmente significativo $(\mathrm{p}=0,053)$ a pesar de los ajustes hechos para mantener los grupos de edad lo más homogéneos posibles.

Nuestros resultados pueden diferir de otros por dos consideraciones importantes: la primera es el haber considerado para este análisis únicamente los pacientes que ingresaron con IRA al hospital, es decir la IRA adquirida en la comunidad, por lo que en este estudio no están incluidos los pacientes que desarrollan IRA dentro del hospital por efecto de las intervenciones médicas o los procesos mórbidos intercurrentes y que corresponden a un universo diferente. Una segunda consideración es la relativa a la población de procedencia de todos los pacientes; esto es, son pacientes procedentes de un área urbana marginal de Lima Metropolitana, población con características peculiares de demanda basada en dos conceptos: su demanda de servicios sanitarios es extremadamente tardía sobre todo en la población de adultos y más aún de adultos mayores (19) y los pacientes en una proporción considerable solicitan cuidados sobre sus síntomas en lugares de atención sanitaria no convencional como las farmacias, los curanderos u otros basados más en creencias y tradiciones culturales ancestrales, algunas de las cuales basan sus recomendaciones en restricciones de aportes esenciales 
energéticos o no reconocimiento de las intervenciones de antibióticoterapia oportuna y adecuada ante eventos infecciosos intercurrentes o de hidratación insuficiente o inadecuada entre otros $(20,21)$.

Este estudio invita a reflexionar sobre lo relevante de la intervención sanitaria preventiva en ciertas comunidades de nuestra sociedad, intervenciones que debieran sustentarse en la educación, la consejería y la intervención del cuidado de las familias extra hospitalariamente respetando su tradición y cultura.

En conclusión, nuestro estudio permite decir que la IRA por sí misma no tiene efecto en la mortalidad en el largo plazo y que ésta, está mayormente relacionada a las características de vulnerabilidad biológica de los pacientes: edad muy avanzada, desnutrición y coexistencia de neoplasia maligna.

\section{Declaración de financiamiento y de conflictos de intereses:}

El trabajo ha sido financiado por los mismos autores. Además, los autores declaran que no existen conflictos de interés con respecto a la publicación de este artículo.

\section{Contribución de autoría:}

JC: Concepción y diseño del estudio, análisis e interpretación de los resultados, aprobación de la versión para publicación; JLL, AC: Concepción y diseño del estudio, recopilación de los datos, análisis e interpretación de los resultados.

\section{Correspondencia:}

Augusto Xavier Cama Olivares

Jr Cesar Vallejo 1051 Covida - Los Olivos. Lima, Perú.

Correo electrónico: augusto.cama.o@upch.pe

Teléfono: 51994996386

\section{REFERENCIAS BIBLIOGRÁFICAS}

1. Miyahira J. Insuficiencia renal aguda. Rev Med Hered. 2003; 14(1):36-43.

2. León C, Cieza J, Valenzuela R. Injuria renal aguda, perfil epidemiológico intrahospitalario y factores asociados al riesgo de muerte. Rev Med Hered. 2014; 25(4):189-95.

3. Lluncor J, Cruz-Encarnación MJ, Cieza J. Factores asociados a injuria renal aguda en pacientes incidentes de un hospital general de Lima- Perú. Rev Med Hered. 2015; 26(1):24-30.
4. Medve L, Antek C, Paloczi B, Kocsi S, Gartner B, Marjanek Z, et al. Epidemiology of acute kidney injury in Hungarian intensive care units: a multicenter, prospective, observational study. BMC Nephrol. 2011; 12:43.

5. Waikar SS, Liu KD, Chertow GM. Diagnosis, epidemiology and outcomes of acute kidney injury. Clin J Am Soc Nephrol. 2008; 3(3):844-61.

6. Mendoza E. Insuficiencia renal aguda en el Hospital Arzobispo Loayza. Tesis presentada para la obtención del título de Médico Cirujano. Lima, Perú: Universidad Peruana Cayetano Heredia; 1993.

7. Cieza J, Gutierrez R, Estremadoiro L, Miyahira J. Insuficiencia renal aguda secundaria a cólera: aspectos epidemiologicos. Rev Inst Méd Sucre. 1994; 59(104):16-23.

8. Santos ER, Matos JD. Perfil epidemiológico dos pacientes com injúria renal aguda em uma Unidade de Terapia Intensiva. ACM Arq Catarin Med. 2008; 37(4):7-11.

9. Hernández JG. Indice clínico pronóstico en pacientes con insuficiencia renal aguda secundaria a enfermedad diarreica coleriforme sometidos a hemodiálisis. Tesis de Maestría. Lima, Perú: Universidad Peruana Cayetano Heredia;1996.

10. Rodríguez VM. Estudio clínico epidemiológico de insuficiencia renal aguda (IRA) en el Hospital Arzobispo Loayza entre los años de 1995 al 2000. Tesis presentada para la obtención del título de Médico Cirujano. Lima, Perú: Universidad Peruana Cayetano Heredia; 2001.

11. Finlay S, Bray B, Lewington AJ, et al. Identification of risk factors associated with acute kidney injury in patients admitted to acute medical units. Clin Med Lond Engl. 2013; 13(3):233-8.

12. Miroslaba G, Vázquez A, Fernández Y, Guerra G. Comportamiento de factores pronósticos de morbilidad y mortalidad en una Unidad de Hemodiálisis. Rev Cuba Med. 2008; 47(3):0-0.

13. Mehta RL, Kellum JA, Shah SV, et al. Acute Kidney Injury Network: report of an initiative to improve outcomes in acute kidney injury. Crit Care Lond Engl. 2007; 11(2):R31.

14. Sovero YE. Cambios del medio interno y de la función renal en ancianos y adultos jóvenes con deshidratación severa por enfermedad diarreica aguda compatible con cólera. Tesis presentada para la obtención del título de Médico Cirujano. Lima, Perú: Universidad Peruana Cayetano Heredia; 1993.

15. Rodríguez V, Navarro B, Gonzaléz M. Respuesta inflamatoria en niños con desnutrición aguda grave y anemia. Perspect Nut Hum. 2008; 10(2):131-142.

16. Álvarez-Dongo D, Sánchez-Abanto J, GómezGuizado G, Tarqui-Mamani C. Sobrepeso y obesidad: prevalencia y determinantes sociales del exceso de peso en la población peruana (2009-2010). Rev Peru 
Med Exp Salud Pública. 2012; 29(3):303-13.

17. Lancheros-Páez LL, Merchán-Chaverra RA, Martínez-Anaya LJ. Screening nutritional risk in cancer patients. Rev Fac Med. 2014; 62:57-64.

18. Escamilla DM, Jarrett P. The impact of weight loss on patients with cancer. Nurs Times. 2016; 112(11):202.

19. Wagstaff A. Pobreza y desigualdades en el sector de la salud. Rev Panam Salud Pública. 2002; 11(56):316-26.
20. Lovera A, Fuentes D. Identification of activities related to the practice and type of complementary and alternative medicine in Lima and Callao (Perú). Vitae. 2013; 20(2):118-24.

21. Peña A, Paco O. Medicina alternativa: intento de análisis. An Fac Med. 2013; 68(1):87-96.

Recibido: 09/01/2017

Aceptado: 23/03/2017 